\title{
Manchester Ovoid
}

National Cancer Institute

\section{Source}

National Cancer Institute. Manchester Ovoid. NCI Thesaurus. Code C106075.

One of a pair of pre-loaded, ovoid-shaped applicators containing radium that is inserted into the vagina as part of the Manchester system for gynecologic intracavity brachytherapy delivery. 